\title{
Network design with edge-connectivity and degree constraints*
}

\author{
Takuro Fukunaga Hiroshi Nagamochi \\ \{takuro, nag\}@amp.i.kyoto-u.ac.jp \\ Department of Applied Mathematics and Physics, \\ Graduate School of Informatics, Kyoto University, Japan
}

\begin{abstract}
We consider the following network design problem; Given a vertex set $V$ with a metric $\operatorname{cost} c$ on $V$, an integer $k \geq 1$, and a degree specification $b$, find a minimum cost $k$-edge-connected multigraph on $V$ under the constraint that the degree of each vertex $v \in V$ is equal to $b(v)$. This problem generalizes metric TSP. In this paper, we propose that the problem admits a $\rho$-approximation algorithm if $b(v) \geq 2, v \in V$, where $\rho=2.5$ if $k$ is even, and $\rho=2.5+1.5 / k$ if $k$ is odd. We also prove that the digraph version of this problem admits a 2.5 -approximation algorithm and discuss some generalization of metric TSP.
\end{abstract}

Keywords: approximation algorithm, degree constraint, edge-connectivity, $(m, n)-$ VRP, TSP, vehicle routing problem

\section{Introduction}

It is a main concern in the field of network design to construct a graph of the least cost which satisfies some connectivity requirement. Actually many results on this topic have been obtained so far. In this paper, we consider a network design problem that asks to find a minimum cost $k$-edge-connected multipraph on a metric edge cost under degree specification. This provides a natural and flexible framework for treating many network design problems. For example, it generalizes the vehicle routing problem with $m$ vehicles ( $m$-VRP) $[4,8]$, which will be introduced below, and hence contains a well-known metric traveling salesperson problem (TSP), which has already been applied to numerous practical problems [9].

Let $\mathbb{Z}_{+}$and $\mathbb{Q}_{+}$denote the sets of non-negative integers and non-negative rational numbers, respectively. Let $G=(V, E)$ be a multigraph with a vertex set $V$ and an edge set $E$, where a multigraph may have some parallel edges but is not allowed to have any loops. For two vertices $u$ and $v$, an edge joining $u$ and $v$ is denoted by $u v$. Since we consider multigraphs in this paper, we distinguish two parallel edges $e_{1}=u v$ and $e_{2}=u v$, which may be simply denoted by $u v$ and $u v$. For a non-empty vertex set $X \subset V$,

\footnotetext{
${ }^{*}$ Technical report 2006-012, October 4, 2006.
} 


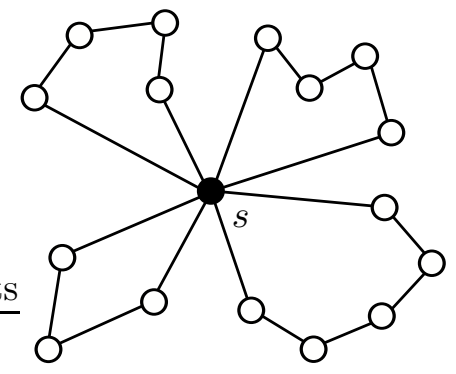

Figure 1: A solution for 4-VRP

$d(X ; G)$ (or $d(X)$ ) denotes the number of edges whose one end vertex is in $X$ and the other is in $V-X$. In particular $d(v ; G)$ (or $d(v)$ ) denotes the degree of vertex $v$ in $G$. The edge-connectivity $\lambda(u, v ; G)$ (or $\lambda(u, v)$ ) between $u$ and $v$ is the maximum number of edge-disjoint paths between them in $G$. The edge-connectivity $\lambda(G)$ of $G$ is defined as $\min _{u, v \in V} \lambda(u, v ; G)$. If $\lambda(G) \geq k$ for some $k \in \mathbb{Z}_{+}$, then $G$ is called $k$-edge-connected. For a function $r:\left(\begin{array}{c}V \\ 2\end{array}\right) \rightarrow \mathbb{Z}_{+}, G$ is called $r$-edge-connected if $\lambda(u, v ; G) \geq r(u, v)$ for every $u, v \in V$. Edge cost $c:\left(\begin{array}{c}V \\ 2\end{array}\right) \rightarrow \mathbb{Q}_{+}$is called metric if it obeys the triangle inequality, i.e., $c(u v)+c(v w) \geq c(u w)$ for every $u, v, w \in V$.

For a degree specification $b: V \rightarrow \mathbb{Z}_{+}$, a multigraph $G$ with $d(v ; G)=b(v)$ for all $v \in V$ is called a perfect b-matching. In this paper, we focus on the following network design problem.

\section{$k$-edge-connected multigraph with degree specification ( $k$-ECMDS):}

A vertex set $V$, a metric edge cost $c:\left(\begin{array}{c}V \\ 2\end{array}\right) \rightarrow \mathbb{Q}_{+}$, a degree specification $b: V \rightarrow \mathbb{Z}_{+}$, and a positive integer $k$ are given. We are asked to find a minimum cost perfect $b$-matching $G=(V, E)$ of edge-connectivity $k$.

In this paper, we suppose that $b(v) \geq 2$ for all $v \in V$ unless stated otherwise, and propose approximation algorithms to $k$-ECMDS in this case.

Problem $k$-ECMDS is a generalization of $m$-VRP, which asks to find a minimum cost set of $m$ cycles, each containing a designated initial city $s$, such that each of the other cities is covered by exactly one cycle (see Fig. 1). Observe that this problem is 2-ECMDS where $b(s)=2 m$ for the initial city $s \in V$ and $b(v)=2$ for every $v \in V-s$. If $m=1$, then $m$-VRP is exactly TSP. Since TSP is known to be NP-hard [12] even if a given cost is metric (metric TSP), $k$-ECMDS is also NP-hard. If a given cost is not metric, TSP cannot be approximated unless $\mathrm{P}=\mathrm{NP}[12]$. For $m$-VRP, there is a 2-approximation algorithm based on the primal-dual method [8].

It is well studied to find a minimum cost multigraph either with $k$-edge-connectivity or with degree specification. It is known that finding a minimum cost $k$-edge-connected graph is NP-hard since it is equivalent to metric TSP when $k=2$ and a given edge cost is metric. On the other hand, it is known that a minimum cost perfect $b$-matching can be constructed in polynomial time (for example, see [11]). As a prior result on problems equipped with both edge-connectivity requirements and degree constraints, Frank [2] showed that it is polynomially solvable to find a minimum cost $r$-edge-connected multigraph $G$ with 
$\ell(v) \leq d(v ; G) \leq u(v), v \in V$ for degree lower and upper bounds $\ell, u: V \rightarrow \mathbb{Z}_{+}$and a metric edge cost $c$ such that $c(u v)$ is defined by $w(u)+w(v)$ for some weight $w: V \rightarrow \mathbb{Q}_{+}$ (in particular, $c(u v)=1$ for every $u v \in\left(\begin{array}{c}V \\ 2\end{array}\right)$ ). Recently Fukunaga and Nagamochi [5] presented approximation algorithms for a network design problem with a general metric edge cost and some degree bounds; For example, they presented a $\left(2+1 /\left\lfloor\min _{u, v \in V} r(u, v) / 2\right\rfloor\right)$ approximation algorithm for constructing a minimum cost $r$-edge-connected multigraph that meets a local-edge-connectivity requirement $r$ with $r(u, v) \geq 2, u, v \in V$ under a uniform degree upper bound. Afterwards Fukunaga and Nagamochi [6] gave a 3approximation algorithm for the case where $r(u, v) \in\{1,2\}$ for every $u, v \in V$ and $\ell(v)=u(v)$ for each $v \in V$. In this paper, we extend the 3-approximation result [6] to $k$-ECMDS. Concretely, we prove that $k$-ECMDS is $\rho$-approximable if $b(v) \geq 2, v \in V$, where $\rho=2.5$ if $k$ is even and $\rho=2.5+1.5 / k$ if $k$ is odd. Moreover, we show that this factor can be improved when a degree specification is uniform. To design our algorithms for $k$-ECMDS, we take a similar approach with famous 2- and 1.5-approximation algorithms for metric TSP.

Furthermore, we also generalize $k$-ECMDS to a network design problem in digraphs. We denote an arc (i.e., a directed edge) from a vertex $u$ to another vertex $v$ by $u v$. Two $\operatorname{arcs}$ from $u$ to $v$ are called parallel. Let $D=(V, A)$ be a multi-digraph, where a multi-digraph may have some parallel arcs but is not allowed to have any loops. For an ordered pair of vertices $u$ and $v, \lambda(u, v ; D)$ (or $\lambda(u, v)$ ) denotes the arc-connectivity from $u$ to $v$, i.e., the maximum number of arc-disjoint paths from $u$ to $v$ in $D$. The arc-connectivity $\lambda(D)$ of $D$ is defined as $\min _{u, v \in V} \lambda(u, v ; D)$. If $\lambda(D) \geq k$ for some $k \in \mathbb{Z}_{+}, D$ is called $k$-arc-connected. Moreover, $d^{-}(v ; D)$ (or $\left.d^{-}(v)\right)$ and $d^{+}(v ; D)$ (or $d^{+}(v)$ ) denote in- and out-degree of vertex $v$ in digraph $D$, respectively. Arc cost $c: V \times V \rightarrow \mathbb{Q}_{+}$is called symmetric if $c(u v)=c(v u)$ for every $u, v \in V$, and metric if it obeys the triangle inequality, i.e., $c(u v)+c(v z) \geq c(u z)$ for every $u, v, z \in V$.

We call a multi-digraph $D$ with $d^{-}(v ; D)=b^{-}(v)$ and $d^{+}(v ; D)=b^{+}(v)$ for all $v \in$ $V$ perfect $\left(b^{-}, b^{+}\right)$-matching for in- and out-degree specifications $b^{-}, b^{+}: V \rightarrow \mathbb{Z}_{+}$. A minimum cost perfect $\left(b^{-}, b^{+}\right)$-matching can be found by computing a minimum cost perfect $b$-matching in a bipartite graph. The digraph version of the problem is described as follows.

\section{$k$-arc-connected multi-digraph with degree specification ( $k$-ACMDS):}

A vertex set $V$, a symmetric metric arc cost $c: V \times V \rightarrow \mathbb{Q}_{+}$, in- and out-degree specifications $b^{-}, b^{+}: V \rightarrow \mathbb{Z}_{+}$, and a positive integer $k$ are given. We are asked to find a minimum cost perfect $\left(b^{-}, b^{+}\right)$-matching $D=(V, A)$ of arc-connectivity $k$.

We also introduce a problem $(m, n)$-vehicle routing problem $((m, n)$-VRP), which generalizes $m$-VRP so that each of the other cities than a special city is visited by exactly $n$ of the $m$ cycles. This problem is not contained in $k$-ECMDS. However, we show that our algorithm for $k$-ECMDS also delivers a 2.5-approximate solution to $(m, n)$-VRP. Moreover, we improve this algorithm to an $\left(1.5+\frac{m-n}{m}\right)$-approximation algorithm.

This paper is organized as follows. Section 2 presents an algorithm for $k$-ECMDS. Section 3 provides a 2.5-approximation algorithm for $k$-ACMDS problem. Section 4 im- 
proves the approximation factors of these algorithms assuming that a degree specification is uniform. Section 5 shows how to apply our algorithm for $k$-ECMDS to $(m, n)$-VRP. Section 6 makes some concluding remarks.

\section{Algorithm for $k$-ECMDS}

This section describes an approximation algorithm for $k$-ECMDS. Before describing the algorithm, we consider how to check the feasibility of a given instance.

\section{$2.1 \quad$ Feasibility}

For some degree specification $b$, there is no perfect $b$-matching. The following theorem shows provides a necessary and sufficient condition for a degree specification to admit a perfect $b$-matching. Note that $b(v)$ can be 1 in this theorem.

Theorem 1 Let $V$ be a vertex set with $|V| \geq 2$ and $b: V \rightarrow \mathbb{Z}_{+}$be a degree specification. Then there exists a perfect b-matching if and only if $\sum_{v \in V} b(v)$ is even and $b(v) \leq \sum_{u \in V-v} b(u)$ for each $v \in V$.

Proof: The necessity is trivial. We show the sufficiency by constructing a perfect $b$ matching. We let $V=\left\{v_{1}, \ldots, v_{n}\right\}$ and $B=\sum_{\ell=1}^{n} b\left(v_{\ell}\right) / 2$. For $j=1, \ldots, B$, we define $i_{j}$ as the minimum integer such that $\sum_{\ell=1}^{i_{j}} b\left(v_{\ell}\right) \geq j$, and $i_{j}^{\prime}$ as the minimum integer such that

$\sum_{\ell=1}^{i_{j}^{\prime}} b\left(v_{\ell}\right) \geq B+j$. Notice that $\sum_{\ell=1}^{i_{j}-1} b\left(v_{\ell}\right)<j$ holds by the definition if $i_{j} \geq 2$. Then we can see that $i_{j} \neq i_{j}^{\prime}$ since otherwise we would have $b\left(v_{i_{j}}\right)=\sum_{\ell=1}^{i_{j}} b\left(v_{\ell}\right)-\sum_{\ell=1}^{i_{j}-1} b\left(v_{\ell}\right)>$ $(B+j)-j=B$ if $i_{j} \geq 2$ and $b\left(v_{i_{j}}\right) \geq B+j>B$ otherwise, which contradicts to the assumption.

Let $M=\left\{e_{j}=v_{i_{j}} v_{i_{j}^{\prime}} \mid j=1, \ldots, B\right\}$. Then $M$ contains no loop by $i_{j} \neq i_{j}^{\prime}$. Moreover $G_{M}$ is a perfect $b$-matching since $\mid\left\{j \mid i_{j}=\ell\right.$ or $\left.i_{j}^{\prime}=\ell\right\} \mid=b\left(v_{i}\right)$, as required.

Theorem 1 does not mention the edge-connectivity. For existence of connected perfect $b$-matchings, we additionally need the condition that $\sum_{v \in V} b(v) \geq 2(|V|-1)$ [6]. This is always satisfied if $b(v) \geq 2, v \in V$, which we assume for 1-ECMDS. For $k \geq 2$, the conditions in Theorem 1 and $b(v) \geq k, v \in V$ are sufficient for the existence of $k$-edgeconnected perfect $b$-matchings as our algorithm will construct such $b$-matchings under the conditions.

\subsection{Algorithm}

Now we describe our algorithm to $k$-ECMDS. Let $(V, b, c, k)$ be an instance of $k$-ECMDS. The conditions appeared in Theorem 1 and $b(v) \geq k$ for all $v \in V$ can be verified in polynomial time, where they are apparently necessary for an instance to have $k$-edgeconnected perfect $b$-matchings. Hence our algorithm checks them, and if some of them are violated, it outputs message "INFEASIBLE". In the following, we suppose the existence of perfect $b$-matchings with $b(v) \geq k$ for all $v \in V$. If $2 \leq|V| \leq 3$, then every perfect $b$-matching is $k$-edge-connected because any non-empty vertex set $X \subset V$ is $\{v\}$ or $V-\{v\}$ 
for some $v \in V$, and then $d(X)=d(v) \geq k$. Hence we can assume without loss of generality that $|V| \geq 4$.

For an edge set $F$ on $V$, we denote graph $(V, F)$ by $G_{F}$. Let $M$ be a minimum cost edge set such that $G_{M}$ is a perfect $b$-matching. In addition, let $H$ be an edge set of a Hamiltonian cycle spanning $V$ constructed by the 1.5-approximation algorithm for TSP due to Christofides [12].

Initialization: After testing the feasibility of a given instance, our algorithm first prepares $M$ and $k^{\prime}=\lceil k / 2\rceil$ copies $H_{1}, \ldots, H_{k^{\prime}}$ of $H$. Let $E$ denote the union $M \cup H_{1} \cup \cdots \cup$ $H_{k^{\prime}}$ of them. Notice that $G_{E}$ is $2 k^{\prime}$-edge-connected by the existence of edge-disjoint $k^{\prime}$ Hamiltonian cycles. We call a vertex $v$ in a handling graph $G$ an excess vertex if $d(v ; G)>b(v)$ (otherwise a non-excess vertex). In $G_{E}$, all vertices are excess vertices since $d\left(v ; G_{E}\right)=b(v)+2 k^{\prime}$. In the following steps, the algorithm reduces the degree of excess vertices until no excess vertex exists while generating no loops and keeping $k$-edgeconnectivity (Notice that $k<2 k^{\prime}$ if $k$ is odd). This is achieved by two phases, Phase 1 and Phase 2, as follows.

Phase 1: In this phase, we modify only edges in $M$ while keeping edges in $H_{1}, \ldots, H_{k^{\prime}}$ unchanged. We define the following two operations on an excess vertex $v \in V$.

Operation 1: If $v$ has two incident edges $x v$ and $y v$ in $M$ with $x \neq y$, replace $x v$ and $y v$ by new edge $x y$.

Operation 2: If $v$ has two parallel edges $u v$ in $M$ with $d(u)>b(u)$, remove those edges.

Phase 1 repeats Operations 1 and 2 until none of them is executable. For avoiding ambiguity, we let $M^{\prime}$ denote $M$ after executing Phase 1, and $M$ denote the original set in what follows. Moreover, let $E^{\prime}=M^{\prime} \cup H_{1} \cup \cdots \cup H_{k^{\prime}}$. Note that $d(v)-b(v)$ is always a nonnegative even integer throughout (and after) these operations because $d\left(v ; G_{E}\right)-b(v)=2 k^{\prime}$ and each operation decreases the degree of a vertex by 2 . If no excess vertex remains in $G_{E^{\prime}}$, then we are done. We consider the case in which there remain some excess vertices, and show some properties on $M^{\prime}$ before describing Phase 2 .

Claim 1 Every excess vertex in $G_{E^{\prime}}$ has at least one incident edge in $M^{\prime}$ and its neighbors in $G_{M^{\prime}}$ are unique.

Proof: Since $d\left(v ; G_{E^{\prime}}\right)-b(v)$ is a positive even integer for an excess vertex $v$ in $G_{E^{\prime}}$, it holds $d\left(v ; G_{M^{\prime}}\right)=d\left(v ; G_{E^{\prime}}\right)-d\left(v ; G_{H_{1} \cup \cdots \cup H_{k^{\prime}}}\right) \geq(b(v)+2)-2 k^{\prime}>0$, Hence $v$ has at least one incident edges in $M^{\prime}$. If neighbors of $v$ in $G_{M^{\prime}}$ are not unique, Operation 1 can be applied to $v$.

For an excess vertex $v$ in $G_{E^{\prime}}$, let $n(v)$ denote the unique neighbor of $v$ in $G_{M^{\prime}}$. If $n(v)$ is also an excess vertex in $G_{E^{\prime}}$, we call the pair $\{v, n(v)\}$ by a strict pair.

Claim 2 Let $\{v, n(v)\}$ be a strict pair. Then $d\left(v ; G_{M^{\prime}}\right)=d\left(n(v) ; G_{M^{\prime}}\right)=1, k$ is odd, and $b(v)=b(n(v))=k$. 
Proof: By Claim $1, d\left(v ; G_{M^{\prime}}\right)=d\left(n(v) ; G_{M^{\prime}}\right)$. If $d\left(v ; G_{M^{\prime}}\right)=d\left(n(v) ; G_{M^{\prime}}\right)>1$, Operation 2 can be applied to $v$ and $n(v)$, a contradiction. Hence $d\left(v ; G_{M^{\prime}}\right)=d\left(n(v) ; G_{M^{\prime}}\right)=1$ holds. Let $u \in\{v, n(v)\}$. Then it holds that $d\left(u ; G_{E^{\prime}}\right)=d\left(u ; G_{H_{1} \cup \ldots \cup H_{k^{\prime}}}\right)+d\left(u ; G_{M^{\prime}}\right)=$ $2 k^{\prime}+1=2\lceil k / 2\rceil+1$. Since $d\left(u ; G_{E^{\prime}}\right)-b(u)$ is even, $b(u)$ must be odd. This fact and $d\left(u, G_{E^{\prime}}\right)>b(u) \geq k$ indicates that $b(u)=k$ and $k$ is odd.

By definition, the existence of excess vertices which are in no strict pairs indicate that of some non-excess vertices. Upon completion of Phase 1, let $N$ denote the set of non-excess vertices in $G_{E^{\prime}}$, and $S$ denote the set of strict pairs in $G_{E^{\prime}}$. If $N=\emptyset$, all excess vertices are in some strict pairs. By Claim 2, $k$ is an odd integer in this case, and furthermore $k \geq 3$ by the assumption that $b(v) \geq 2, v \in V$ if $k=1$. From this fact and $|V| \geq 4, N=\emptyset$ implies that at least two strict pairs exist (i.e., $|S| \geq 2$ ).

Phase 2: Now we describe Phase 2. First, we deal with a special case in which $V$ consists of only two strict pairs.

Claim 3 If $V$ consists of two strict pairs after Phase 1, we can transform $G_{E^{\prime}}$ into a $k$-edge-connected perfect b-matching without increasing the cost.

Proof: Let $V=\{u, v, w, z\}$ and $H=\{u v, v w, w z, z u\}$. Now $E^{\prime}=M^{\prime} \cup H_{1} \cup \cdots \cup H_{k^{\prime}}$ $(k \geq 2)$. Then either $M^{\prime}=\{u v, w z\}$ (or $\left.\{v w, z u\}\right)$ or $M^{\prime}=\{u w, v z\}$ holds. In both cases, we replace $M^{\prime} \cup H_{1} \cup H_{2}$ by $E^{\prime \prime}=\{u v, v w, w z, z u, u w, v z\}$ (see Fig. 2). Then, we can see that $d\left(v ; G_{E^{\prime \prime}}\right)=3$ for all $v \in V$ and $G_{E^{\prime \prime}}$ is 3-edge-connected. Since $d\left(v ; G_{H_{i}}\right)=$ 2 for $v \in V, i=3, \ldots, k^{\prime}$ and $G_{H_{i}}$ is 2-edge-connected for $i=3, \ldots, k^{\prime}$, it holds that $d\left(v ; G_{E^{\prime \prime} \cup H_{3} \cup \cdots \cup H_{k^{\prime}}}\right)=3+2\left(k^{\prime}-2\right)=k=b(v)$ for $v \in V$ and the edge-connectivity of $G_{E^{\prime \prime} \cup H_{3} \cup \cdots \cup H_{k^{\prime}}}$ is $3+2\left(k^{\prime}-2\right)=k$ (The existence of strict pair implies that $k$ is odd by Claim 2.).

Hence it suffices to show that $c\left(E^{\prime \prime}\right) \leq c\left(M^{\prime}\right)+c\left(H_{1}\right)+c\left(H_{2}\right)$. If $M^{\prime}=\{u w, v z\}$ (or $\{v w, z u\}$ ), then it is obvious since $E^{\prime \prime}=M^{\prime} \cup H_{1} \subseteq M^{\prime} \cup H_{1} \cup H_{2}$. Let us consider the other case, i.e., $M^{\prime}=\{u v, w z\}$. From $M^{\prime} \cup H_{1} \cup H_{2}$, remove $\{u v, u v\}$, replace $\{w z, z u\}$ by $\{w u\}$, and replace $\{v w, w z\}$ by $\{v z\}$. Then the edge set becomes $E^{\prime \prime}$ without increasing edge cost, as required.

In the following, we assume that $|S| \geq 3$ when $N=\emptyset$. In this case, Phase 2 modifies only edges in $H_{i}, i=1, \ldots, k^{\prime}$ while keeping the edges in $M^{\prime}$ unchanged. Let $V\left(H_{i}\right)$ denote the set of vertices spanned by $H_{i}$. We define detaching $v$ from cycle $H_{i}$ to be an operation that replaces the pair $\{u v, v w\} \subseteq H_{i}$ of edges incident to $v$ by a new edge $u w$. Note that this decreases $d(v)$ by 2 , but $H_{i}$ remains a cycle on $V\left(H_{i}\right):=V\left(H_{i}\right)-\{v\}$. For each excess vertex $v$ in $G_{E^{\prime}}$, Phase 2 reduces $d(v)$ to $b(v)$ by detaching $v$ from $\left(d\left(v ; G_{E^{\prime}}\right)-b(v)\right) / 2$ cycles in $H_{1}, \ldots, H_{k^{\prime}}$. We notice that $\left(d\left(v ; G_{E^{\prime}}\right)-b(v)\right) / 2 \leq k^{\prime}$ by $d\left(v ; G_{E^{\prime}}\right)-b(v) \leq$ $d\left(v ; G_{E}\right)-b(v)=2 k^{\prime}$. One important point is to keep $\left|V\left(H_{i}\right)\right| \geq 2$ for each $i=1, \ldots, k^{\prime}$ during Phase 2. In other words, we always select $H_{i}$ with $\left|V\left(H_{i}\right)\right| \geq 3$ to detach an excess vertex. This is necessary because, if we detach a vertex from $H_{i}$ with $V\left(H_{i}\right)=2$, then $H_{i}$ becomes a loop. In addition, we detach the two excess vertices $u$ and $v$ in a strict pair from different cycles in $H_{1}, \ldots, H_{k^{\prime}}$, respectively. This is in order to maintain the $k$-edge-connectivity of $G_{E^{\prime}}$ as will be explained below. 


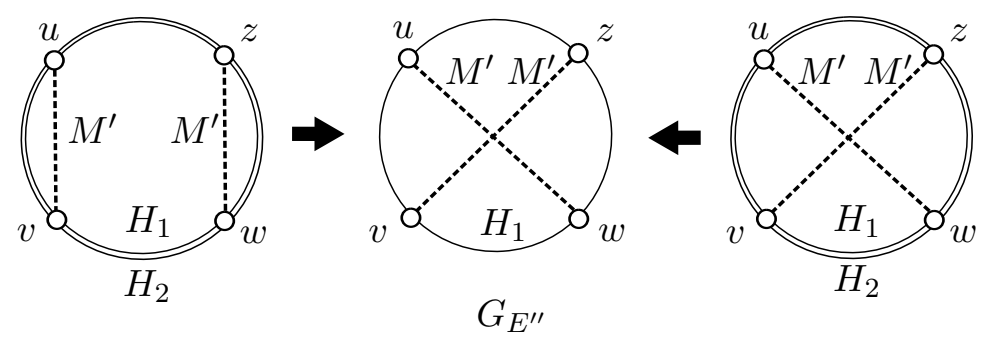

Figure 2: Operations when $V$ consists of two strict pairs

Claim 4 It is possible to decrease the degree of each excess vertex $v$ in $G_{E^{\prime}}$ to $b(v)$ by detaching from some cycles in $H_{1}, \ldots, H_{k^{\prime}}$ so that $\left|V\left(H_{i}\right)\right|$ remains at least 2 for $i=$ $1, \ldots, k^{\prime}$ and the two excess vertices in each strict pair are detached from $H_{i}$ and $H_{j}$ with $i \neq j$, respectively.

Proof: First, let us consider the case of $S \neq \emptyset$. Recall $k \geq 3$ and $k^{\prime}=\lceil k / 2\rceil \geq 2$ in this case. For each strict pair $\{u, v\} \in S$, we detach $u$ and $v$ from different cycles in $H_{1}, \ldots, H_{k^{\prime}}$. On the other hand, we detach excess vertex $z$ from arbitrary $\left(d\left(z ; G_{E^{\prime}}\right)-b(z)\right) / 2$ cycles. After this, each of $H_{1}, \ldots, H_{k^{\prime}}$ is incident to at least one vertex of any strict pair in $S$ in addition to all non-excess vertices in $N$. By the relation between $|S|$ and $|N|$ we explained in the above, it holds that $\left|V\left(H_{i}\right)\right| \geq|S|+|N| \geq 2$ for each $i=1, \ldots, k^{\prime}$, as required.

Next, let us consider the case of $S=\emptyset$. As explained in the above, $|N| \geq 1$ holds for this case. If $|N| \geq 2$, the claim is obvious since each of $H_{1}, \ldots, H_{k^{\prime}}$ is always incident to all vertices in $N$. Hence suppose that $|N|=1$, and let $x$ be the unique non-excess vertex in $N$. Then all edges in $M^{\prime}$ are incident to $x$, since otherwise $S=\emptyset$ implies that Operation 1 or 2 would be applicable to some vertex in $V-x$. In other words, $b(x)=d\left(x ; G_{E^{\prime}}\right)=\left|M^{\prime}\right|+2 k^{\prime}$ holds before Phase 2. Moreover $\sum_{v \in V-x} b(v) \geq b(x)$ also holds by the assumption that perfect $b$-matchings exist. Now assume that we have converted some excess vertices in $G_{E^{\prime}}$ into non-excess vertices by detaching them from some of $H_{1}, \ldots, H_{k^{\prime}}$ while keeping $\left|V\left(H_{i}\right)\right| \geq 2, i=1, \ldots, k^{\prime}$, and yet an excess vertex $y \in V-x$ remains. Hence $\sum_{v \in V} d(v)>\sum_{v \in V} b(v)$. Then there remains a cycle $H_{i}$ with $\left|V\left(H_{i}\right)\right|>2$ because

$$
\begin{aligned}
2 \sum_{1 \leq i \leq k^{\prime}}\left|V\left(H_{i}\right)\right|=\sum_{v \in V} d\left(v ; G_{H_{1} \cup \cdots \cup H_{k^{\prime}}}\right)=\sum_{v \in V} d(v)-2\left|M^{\prime}\right| \\
\quad>\sum_{v \in V-\{x\}} b(v)+b(x)-2\left|M^{\prime}\right| \geq 2\left(b(x)-\left|M^{\prime}\right|\right) \geq 4 k^{\prime} .
\end{aligned}
$$

Therefore we can detach an excess vertex $y$ from such $H_{i}$ as long as such a vertex exists. This implies that the claim holds also for $|N|=1$.

In the following, we let $H_{i}^{\prime}$ denote $H_{i}$ after Phase 2 , and $H_{i}$ denote the original Hamiltonian cycle for $i=1, \ldots, k^{\prime}$. Moreover let $E^{\prime \prime}=M^{\prime} \cup H_{1}^{\prime} \cup \cdots \cup H_{k^{\prime}}^{\prime}$. The algorithm outputs $G_{E^{\prime \prime}}$. The entire algorithm is described as follows.

Algorithm UNDIRECT $(k)$ 
Input: A vertex set $V$, a degree specification $b: V \rightarrow \mathbb{Z}_{+}$, a metric edge cost $c: V \rightarrow \mathbb{Q}_{+}$, and a positive integer $k$

Output: A $k$-edge-connected perfect $b$-matching or "INFEASIBLE"

1: if $\sum_{v \in V} b(v)$ is odd, $\exists v: b(v)>\sum_{u \in V-v} b(u)$ or $k>b(v)$ then

2: Output "INFEASIBLE" and halt

3: end if;

4: Compute a minimum cost perfect $b$-matching $G_{M}$;

5: if $|V| \leq 3$ then

6: $\quad$ Output $G_{M}$ and halt

7: end if;

8: Compute a Hamiltonian cycle $G_{H}$ on $V$ by Christofides' algorithm;

9: $k^{\prime}:=\lceil k / 2\rceil$; Let $H_{1}, \ldots, H_{k^{\prime}}$ be $k^{\prime}$ copies of $H$;

\# Phase 1

10: $M^{\prime}:=M$;

11: while Operation 1 or 2 is applicable to a vertex $v \in V$

with $d\left(v ; G_{M^{\prime} \cup H_{1} \cup \cdots \cup H_{k^{\prime}}}\right)>b(v)$ do

12: $\quad$ if $\exists\{x v, v y\} \subseteq M^{\prime}$ such that $x \neq y$ then

13: $\quad M^{\prime}:=\left(M^{\prime}-\{x v, v y\}\right) \cup\{x y\} \quad$ \# Operation 1

14: $\quad$ else

15: $\quad$ if $\exists\{x v, v x\} \subseteq M^{\prime}$ such that $d\left(x ; G_{M^{\prime} \cup H_{1} \cup \cdots \cup H_{k^{\prime}}}\right)>b(x)$ then

16:

17:

18:

$$
M^{\prime}:=M^{\prime}-\{x v, v x\} \quad \text { \# Operation } 2
$$

end if

\section{end if}

19: end while;

\# Phase 2

20: if $V$ consists of two strict pairs then

21: $\quad$ Rename vertices so that $H=\{u v, v w, w z, z u\}$;

22: $\quad H_{2}^{\prime}:=\emptyset ; M^{\prime}:=\{u w, v z\}$;

23: Output $G_{M^{\prime} \cup H_{1}^{\prime} \cup \cdots \cup H_{k^{\prime}}^{\prime}}$ and halt

24: end if;

25: $H_{i}^{\prime}:=H_{i}$ for each $i=1, \ldots, k^{\prime}$;

26: while $\exists v \in V$ with $d\left(v ; G_{M^{\prime} \cup H_{1}^{\prime} \cup \ldots \cup H_{k^{\prime}}^{\prime}}\right)>b(v)$ do

27: $\quad$ if $v$ and $n(v)$ forms a strict pair then

28: $\quad$ Detach $v$ from $H_{i}^{\prime}$ and $n(v)$ from $H_{j}^{\prime}$, where $i \neq j$

29: else

30: $\quad$ Detach $v$ from $H_{i}^{\prime}$ with $V\left(H_{i}^{\prime}\right)>2$

31: $\quad$ end if

32: end while;

33: $E^{\prime \prime}:=M^{\prime} \cup H_{1}^{\prime} \cup \cdots \cup H_{k^{\prime}}^{\prime}$;

34: Output $G_{E^{\prime \prime}}$

Claim $5 G_{E^{\prime \prime}}$ is a $k$-edge-connected perfect b-matching. 


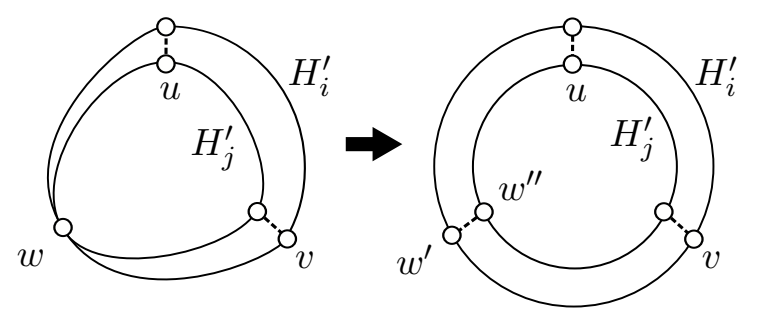

(a)

(b)

Figure 3: Reduction to the case of $V\left(H_{i}^{\prime}\right) \cap V\left(H_{j}^{\prime}\right)=\emptyset$

Proof: We have already seen the case in which $V$ consists of two strict pairs. Hence we suppose the other case in the following. Moreover we have already observed that $d\left(v ; G_{E^{\prime \prime}}\right)=b(v)$ holds for each $v \in V$. Furthermore $G_{E^{\prime \prime}}$ is loopless since $G_{E}$ is loopless and no operations in the algorithm generate loops. Hence we prove the $k$-edge-connectivity of $G_{E^{\prime \prime}}$ below.

Let $u, v \in V$. (i) First suppose that $u$ and $v$ are in some (possibly different) strict pairs in $G_{E^{\prime}}$. Moreover, let $u \notin V\left(H_{i}^{\prime}\right)$ and $v \notin V\left(H_{j}^{\prime}\right)$ (hence $u \in V\left(H_{i^{\prime}}^{\prime}\right)$ for $i^{\prime} \neq i$ and $v \in V\left(H_{j^{\prime}}^{\prime}\right)$ for $\left.j^{\prime} \neq j\right)$. For each $\ell \in\left\{1, \ldots, k^{\prime}\right\}-\{i, j\}, \lambda\left(u, v ; G_{H_{\ell}^{\prime}}\right)=2$ holds because $u, v \in V\left(H_{\ell}^{\prime}\right)$. If $i=j, \lambda\left(u, v ; G_{H_{i}^{\prime} \cup M^{\prime}}\right)=1$ holds because $d\left(u ; G_{M^{\prime}}\right)=d\left(v ; G_{M^{\prime}}\right)=1$ and $n(u), n(v) \in V\left(H_{i}^{\prime}\right)$. Then it holds that $\lambda\left(u, v ; G_{E^{\prime \prime}}\right)=2\left(k^{\prime}-1\right)+1=k$ in this case (Recall that the existence of strict pairs implies that $k$ is odd by Claim 2). Hence we let $i \neq j$, and show that $\lambda\left(u, v ; G_{H_{i}^{\prime} \cup H_{j}^{\prime} \cup M^{\prime}}\right) \geq 3$ from now on, from which $\lambda\left(u, v ; G_{E^{\prime \prime}}\right) \geq 2\left(k^{\prime}-2\right)+3=k$ can be derived.

Let $N$ and $S$ denote the sets of non-excess vertices and strict pairs in $G_{E^{\prime}}$ after Phase 1, respectively. Suppose that $V\left(H_{i}^{\prime}\right) \cap V\left(H_{j}^{\prime}\right)=\emptyset$. In this case, it can be seen that $N=\emptyset$, and hence $|S| \geq 3$ by the assumption about the relation between $N$ and $S$. Since at least one vertex of each strict pair is spanned by each cycle in $H_{1}^{\prime}, \ldots, H_{k^{\prime}}^{\prime}$, we can see that $M^{\prime}$ contains at least three vertex-disjoint edges that join vertices in $V\left(H_{i}^{\prime}\right)$ and in $V\left(H_{j}^{\prime}\right)$, two of which are $u$ and $v$. This indicates that $\lambda\left(u, v ; G_{H_{i}^{\prime} \cup H_{j}^{\prime} \cup M^{\prime}}\right) \geq 3$ holds (see the graph of Figure $3(\mathrm{~b})$ ).

Let us consider the case of $V\left(H_{i}^{\prime}\right) \cap V\left(H_{j}^{\prime}\right) \neq \emptyset$ in the next. By the existence of $u$ and $v,|S| \geq 1$ holds. If $u$ and $v$ forms a strict pair (i.e., $\left.u v \in M^{\prime}\right), \lambda\left(u, v ; G_{M^{\prime}}\right)=1$ holds. Since $V\left(H_{i}^{\prime}\right) \cap V\left(H_{j}^{\prime}\right) \neq \emptyset$ implies $\lambda\left(G_{H_{i}^{\prime} \cup H_{j}^{\prime}}\right) \geq 2$, we see that $\lambda\left(u, v ; G_{H_{i}^{\prime} \cup H_{j}^{\prime} \cup M^{\prime}}\right) \geq 3$ in this case. Thus let $u$ and $v$ belong to different strict pairs (i.e., $|S| \geq 2$ ). Then there exists two vertex-disjoint edges in $M^{\prime}$ joins vertices in $V\left(H_{i}^{\prime}\right)$ and in $V\left(H_{j}^{\prime}\right)$ (see Figure 3 (a)). If we split each vertex $w \in V\left(H_{i}^{\prime}\right) \cap V\left(H_{j}^{\prime}\right)$ into two vertices $w^{\prime}$ and $w^{\prime \prime}$ so that $H_{i}^{\prime}$ and $H_{j}^{\prime}$ are vertex-disjoint cycles, and add new edges $w^{\prime} w^{\prime \prime}$ joining those two split vertices to $M^{\prime}$, then we can reduce this case to the case of $V\left(H_{i}^{\prime}\right) \cap V\left(H_{j}^{\prime}\right)=\emptyset$, in which $\lambda\left(u, v ; G_{H_{i}^{\prime} \cup H_{j}^{\prime} \cup M^{\prime}}\right) \geq 3$ has already been observed in the above (see Figure 3 ). Accordingly, we have $\lambda\left(u, v ; G_{H_{i}^{\prime} \cup H_{j}^{\prime} \cup M^{\prime}}\right) \geq 3$ if $u$ and $v$ are in some strict pairs, as required.

(ii) In the next, let $u$ and $v$ be not in any strict pairs. For $z \in\{u, v\}$, let $n^{\prime}(z)$ denote $z$ itself if $z \in N$, and $n(z)$ otherwise. Notice that $n^{\prime}(z) \in N$ for any $z \in\{u, v\}$, i.e., it is spanned by $H_{1}^{\prime}, \ldots, H_{k^{\prime}}^{\prime}$. If $z \in\{u, v\}$ is not spanned by $p>0$ cycles in $H_{1}^{\prime}, \ldots, H_{k^{\prime}}^{\prime}$ (and hence $z$ is an excess vertex in $\left.G_{E^{\prime}}\right)$, then $z$ has at least $k-2\left(k^{\prime}-p\right)$ incident edges in $M^{\prime}$ 
because $d\left(z ; G_{M^{\prime}}\right)=b(z)-d\left(z ; G_{H_{1}^{\prime} \cup \cdots \cup H_{k^{\prime}}^{\prime}}\right) \geq k-2\left(k^{\prime}-p\right)$. Hence $\lambda\left(z, n^{\prime}(z) ; G_{E^{\prime \prime}}\right) \geq$ $2\left(k^{\prime}-p\right)+k-2\left(k^{\prime}-p\right)=k$ holds for each $z \in\{u, v\}$, where we define $\lambda\left(z, z ; G_{E^{\prime \prime}}\right)=+\infty$. Moreover it is obvious that $\lambda\left(n^{\prime}(u), n^{\prime}(v) ; G_{E^{\prime \prime}}\right) \geq 2 k^{\prime}$. Therefore, it holds that

$$
\lambda\left(u, v ; G_{E^{\prime \prime}}\right) \geq \min \left\{\lambda\left(u, n^{\prime}(u) ; G_{E^{\prime \prime}}\right), \lambda\left(n^{\prime}(u), n^{\prime}(v) ; G_{E^{\prime \prime}}\right), \lambda\left(n^{\prime}(v), v ; G_{E^{\prime \prime}}\right)\right\} \geq k .
$$

(iii) Finally, let us consider the remaining case, i.e., $u$ is in a strict pair and $v$ is a vertex which is not in any strict pair. Let us define $n^{\prime}(v)$ as in the above. Then $\lambda\left(v, n^{\prime}(v) ; G_{E^{\prime \prime}}\right) \geq$ $k$ holds. Without loss of generality, let $u$ be detached from $H_{1}^{\prime}$, and spanned by $H_{2}^{\prime}, \ldots, H_{k^{\prime}}^{\prime}$. Since $u n(u) \in M^{\prime}$ and $n(u), n^{\prime}(v) \in V\left(H_{1}^{\prime}\right)$, it holds that $\lambda\left(u, n(u) ; G_{M^{\prime} \cup H_{1}^{\prime}}\right)=1$, and $\lambda\left(n(u), n^{\prime}(v) ; G_{M^{\prime} \cup H_{1}^{\prime}}\right) \geq 2$. Then,

$$
\begin{aligned}
\lambda\left(u, n^{\prime}(v) ; G_{E^{\prime \prime}}\right) \geq \min \{\lambda(u, & \left.\left.n(u) ; G_{M^{\prime} \cup H_{1}^{\prime}}\right), \lambda\left(n(u), n^{\prime}(v) ; G_{M^{\prime} \cup H_{1}^{\prime}}\right)\right\} \\
& +\lambda\left(u, n^{\prime}(v) ; G_{H_{2}^{\prime} \cup \cdots \cup H_{k^{\prime}}^{\prime}}\right) \geq 1+2\left(k^{\prime}-1\right)=2 k^{\prime}-1=k .
\end{aligned}
$$

Therefore,

$$
\lambda\left(u, v ; G_{E^{\prime \prime}}\right) \geq \min \left\{\lambda\left(u, n^{\prime}(v) ; G_{E^{\prime \prime}}\right), \lambda\left(v, n^{\prime}(v) ; G_{E^{\prime \prime}}\right)\right\} \geq k,
$$

holds, as required.

Let us consider the cost of the graph $G_{E^{\prime \prime}}$. The following theorem on the Christofides' algorithm gives us an upper bound on $c(H)$. Here, we let $\delta(U)$ denote the set of edges whose one end vertex is in $U$ and the other is in $V-U$ for nonempty $U \subset V$.

Theorem $2([7,13])$ Let

$$
\begin{array}{lll}
O P T_{T S P}=\min & \sum_{e \in E} c(e) x(e) & \\
\text { subject to } & \sum_{e \in \delta(U)} x(e) \geq 2 & \text { for each nonempty } U \subset V, \\
& x(e) \geq 0 & \text { for each } e \in E .
\end{array}
$$

Christofides' algorithm for TSP always outputs a solution of cost at most $1.5 O P T_{T S P}$.

Claim $6 c\left(E^{\prime \prime}\right)$ is at most $1+3\lceil k / 2\rceil / k$ times the optimal cost of $k$-ECMDS.

Proof: No operation in Phases 1 and 2 increases the cost of the graph since the edge cost is metric. Hence it suffices to show that $c\left(M \cup H_{1} \cup \cdots \cup H_{k^{\prime}}\right)$ is at most $(1+3\lceil k / 2\rceil / k) \cdot c(G)$, where $G$ denotes an optimal solution of $k$-ECMDS. Since $G$ is a perfect $b$-matching, $c(M) \leq$ $c(G)$ obviously holds. Thus it suffices to show that $c\left(H_{i}\right) \leq 3 c(G) / k$ for $1 \leq i \leq k^{\prime}$, from which the claim follows.

Let $x_{G}:\left(\begin{array}{c}V \\ 2\end{array}\right) \rightarrow \mathbb{Z}_{+}$be the function such that $x_{G}(u v)$ denotes the number of edges joining $u$ and $v$ in $G$. Since $G$ is $k$-edge-connected, $\sum_{e \in \delta(U)} x_{G}(e) \geq k$ holds for every nonempty $U \subset V$. Hence $2 x_{G} / k$ is feasible for the linear programming in Theorem 2, which means that $\mathrm{OPT}_{T S P} \leq 2 c(G) / k$. By Theorem $2, c\left(H_{i}\right) \leq 1.5 \mathrm{OPT}_{T S P}$. Therefore we have $c\left(H_{i}\right) \leq 3 c(G) / k$, as required.

Claims 5 and 6 establish the next. 
Theorem 3 Algorithm UNDIRECT $(k)$ is a $\rho$-approximation algorithm for $k$-ECMDS, where $\rho=2.5$ if $k$ is even and $\rho=2.5+1.5 / k$ if $k$ is odd.

Algorithm UNDIRECT $(k)$ always outputs a solution for $k \geq 2$ as long as there exists a perfect $b$-matching and $b(v) \geq k$ for all $v \in V$. This fact and Theorem 1 imply the following corollary.

Corollary 1 For $k \geq 2$, there exists a $k$-edge-connected perfect $b$-matching if and only if $\sum_{v \in V} b(v)$ is even and $k \leq b(v) \leq \sum_{u \in V-v} b(u)$ for all $v \in V$.

We close this section with a few remarks. The operations in Phases 1 and 2 are equivalent to a graph transformation called splitting, followed by removing generated loops if any. There are many results on the conditions for splitting to maintain the edgeconnectivity [3, 10]. However, the splittings in these results may generate loops. Hence algorithm UNDIRECT $(k)$ needs to specify a sequence of splitting so that removing loops does not make the degrees lower than the degree specification.

One may consider that a perfect $\left(b-2 k^{\prime}\right)$-matching is more appropriate than a perfect $b$-matching as a building block of our algorithm, since there is no excess vertex for the union of a perfect $\left(b-2 k^{\prime}\right)$-matching and $k^{\prime}$ Hamiltonian cycles. However, there is a degree specification $b$ that admits a perfect $b$-matching, and no perfect $\left(b-2 k^{\prime}\right)$-matching. Furthermore, even if there exits a perfect $\left(b-2 k^{\prime}\right)$-matching, the minimum cost of the perfect $\left(b-2 k^{\prime}\right)$-matching may not be a lower bound on the optimal cost of $k$-ECMDS. Therefore we do not use a perfect $\left(b-2 k^{\prime}\right)$-matching in general case. In Section 4 , we show that a perfect $\left(b-2 k^{\prime}\right)$-matching always exist and its cost can be estimated when a degree specification $b$ is uniform.

\section{Algorithm for $k$-ACMDS}

This section shows that $k$-ACMDS is 2.5 -approximable. The algorithm for $k$-ACMDS can be designed analogously with that for $k$-ECMDS. Before describing the algorithm, we consider the feasibility of $k$-ACMDS.

\subsection{Feasibility}

Frobenius' classic theorem (see [11] for example) tells the relation-ship between the existence of perfect bipartite matchings and the minimum size of vertex covers in bipartite graphs.

Theorem 4 (Frobenius) A bipartite graph $G$ has a perfect matching if and only if each vertex cover has size at least $|V(G)| / 2$.

From this, we can immediately derive a condition for a digraph to have a perfect $\left(b^{-}, b^{+}\right)$-matching.

Theorem 5 Let $V$ be a vertex set, and $b^{-}, b^{+}: V \rightarrow \mathbb{Z}_{+}$be in- and out- degree specifications, respectively. There exists a perfect $\left(b^{-}, b^{+}\right)$-matching if and only if $\sum_{v \in V} b^{-}(v)=$ 
$\sum_{v \in V} b^{+}(v), b^{-}(v) \leq \sum_{u \in V-v} b^{+}(u)$ for each $v \in V$, and $b^{+}(v) \leq \sum_{u \in V-v} b^{-}(u)$ for each $v \in V$.

Proof: The necessity is obvious. Hence we consider the sufficiency in the following. For each $v \in V$, prepare two vertex sets $V_{v}^{-}$and $V_{v}^{+}$corresponding to $v$ such that $\left|V_{v}^{-}\right|=b^{-}(v)$ and $\left|V_{v}^{+}\right|=b^{+}(v)$. Furthermore, let $V^{-}=\cup_{v \in V} V_{v}^{-}, V^{+}=\cup_{v \in V} V_{v}^{+}$, and $E=\left\{u^{-} v^{+} \mid\right.$ $\left.u^{-} \in V_{u}^{-}, v^{+} \in V_{v}^{+}, u \neq v\right\}$. Then a perfect matching in a bipartite graph $\left(V^{-}, V^{+}, E\right)$ corresponds to a perfect $\left(b^{-}, b^{+}\right)$-matching on $V$. So by Theorem 4 , it suffices to show that each vertex cover of $\left(V^{-}, V^{+}, E\right)$ has size at least $\left(\left|V^{-}\right|+\left|V^{+}\right|\right) / 2$.

To the contrary, let us suppose that there exists a vertex cover $C \subset V^{-} \cup V^{+}$of $\left(V^{-}, V^{+}, E\right)$ such that $|C|<\left(\left|V^{-}\right|+\left|V^{+}\right|\right) / 2$ under the assumption in this theorem. Since $\left|V^{-}\right|=\sum_{v \in V} b^{-}(v)=\sum_{v \in V} b^{+}(v)=\left|V^{+}\right|$, it holds that $|C|<\left|V^{-}\right|=\left|V^{+}\right|$. This implies the existence of vertices $x \in V^{-}-C$ and $y \in V^{+}-C$. Let $x$ correspond to $u \in V$ (i.e., $x \in V_{u}^{-}$) and $y$ correspond to $v \in V$ (i.e., $y \in V_{v}^{+}$). If $u \neq v$, there exists an edge $x y \in E$, which is not covered by any vertices in $C$, a contradiction. Hence $u=v$ holds. Then $\cup_{z \in V-v}\left(V_{z}^{-} \cup V_{z}^{+}\right) \subseteq C$ holds. This implies that $|C| \geq \sum_{z \in V-v}\left|V_{z}^{-}\right|+\sum_{z \in V-v}\left|V_{z}^{+}\right|$. Then it holds that

$$
\begin{aligned}
\left(\sum_{v \in V} b^{-}(v)+\sum_{v \in V} b^{+}(v)\right) / 2= & \left(\left|V^{-}\right|+\left|V^{+}\right|\right) / 2>|C| \\
& \geq \sum_{z \in V-v}\left|V_{z}^{-}\right|+\sum_{z \in V-v}\left|V_{z}^{+}\right|=\sum_{z \in V-v} b^{-}(z)+\sum_{z \in V-v} b^{+}(z),
\end{aligned}
$$

implying $b^{-}(v)+b^{+}(v)>\sum_{z \in V-v} b^{-}(z)+\sum_{z \in V-v} b^{+}(z)$. However, this indicates that at least $b^{-}(v)>\sum_{z \in V-v} b^{-}(z)$ or $b^{+}(v)>\sum_{z \in V-v} b^{+}(z)$ holds, contradicting to the assumption.

Notice that the proof of Theorem 5 indicates the reduction of the minimum cost perfect $\left(b^{-}, b^{+}\right)$-matching problem to the minimum cost perfect $b$-matching problem in an undirected bipartite graph.

\subsection{Algorithm}

We are ready to explain the algorithm for $k$-ACMDS. In the following, we assume that $b^{-}(v), b^{+}(v) \geq k$ for each $v \in V$ and a perfect $\left(b^{-}, b^{+}\right)$-matching exists.

Let $M$ be a minimum cost perfect $\left(b^{-}, b^{+}\right)$-matching and $H$ be a directed Hamiltonian cycle constructed by Christofides' algorithm for the edge cost obtained from $c$ by ignoring the direction of arcs (Recall that $c$ is symmetric). Moreover let $H_{1}, \ldots, H_{k}$ be $k$ copies of $H, A=M \cup H_{1} \cup \cdots \cup H_{k}$, and $D_{F}$ denote the digraph $(V, F)$ for an $\operatorname{arc} \operatorname{set} F$. A vertex $v \in V$ is called an excess vertex if $d^{-}(v)>b^{-}(v)$ or $d^{+}(v)>b^{+}(v)$ (otherwise $v$ is called a non-excess vertex). Notice that $d^{-}\left(v ; D_{A}\right)-b^{-}(v)=d^{+}\left(v ; D_{A}\right)-b^{+}(v)$. This condition is maintained throughout the algorithm, i.e., $d^{-}(v)>b^{-}(v)$ is equivalent to $d^{+}(v)>b^{+}(v)$. Our algorithm for $k$-ACMDS decreases the degree of excess vertices as $k$-ECMDS. One difference between algorithms for $k$-ECMDS and for $k$-ACMDS is the definition of Operations 1 and 2. These will be executed for a pair of arcs entering and leaving the same vertex as follows. 
Operation 1: If an excess vertex $v$ has two incident $\operatorname{arcs} x v$ and $v y$ in $M$ with $x \neq y$, replace $x v$ and $v y$ by new edge $x y \in M$.

Operation 2: If an excess vertex $v$ has two arcs $u v$ and $v u$ in $M$ with $d^{-}(u)>b^{-}(u)$ (and $\left.d^{+}(v)>b^{+}(v)\right)$, remove these arcs.

Phase 1 of our algorithm modifies edges in $M$ by repeating Operations 1 and 2 until none of them is executable. We let $M^{\prime}$ denote $M$ after Phase 1 , and $M$ denote the original set in the following. Moreover let $A^{\prime}=M^{\prime} \cup H_{1} \cup \cdots \cup H_{k}$, and $N$ denote the set of non-excess vertices in $D_{A^{\prime}}$. Note that the number of arcs in $M^{\prime}$ entering (resp., leaving) each excess vertices $v$ in $D_{A^{\prime}}$ has $d^{-}\left(v ; D_{A^{\prime}}\right)-k \geq d^{-}\left(v ; D_{A^{\prime}}\right)-b^{-}(v)$ (resp., $\left.d^{-}\left(v ; D_{A^{\prime}}\right)-b^{-}(v)>d^{+}\left(v ; D_{A^{\prime}}\right)-b^{+}(v)\right)$ arcs. The other end vertex of them is unique and in $N$ (i.e., a non-excess vertex in $D_{A^{\prime}}$ ) since otherwise Operation 1 or 2 can be applied to $v$. This situation is simpler than after Phase 2 of $\operatorname{UNDIRECT}(k)$ since no correspondence of strict pairs exists. Notice that $N \neq \emptyset$ always holds here.

Phase 2 of our algorithm for $k$-ACMDS modifies edges in $H_{1}, \ldots, H_{k}$ so as to decrease the degrees of all excess vertices as in $\operatorname{UNDIRECT}(k)$. We repeat detaching each excess vertex from some of $H_{1}, \ldots, H_{k}$, where detaching a vertex $v$ from $H_{i}$ is defined as an operation that replaces the pair $\{u v, v w\} \subseteq H_{i}$ of arcs entering and leaving $v$ by new arc $u w$. We can prove that it is possible to detach excess vertices from Hamiltonian cycles while keeping $V\left(H_{i}\right) \geq 2$ for $1 \leq i \leq k$ as in $\operatorname{UNDIRECT}(k)$.

Claim 7 It is possible to decrease the degree of each excess vertex $v$ to $b(v)$ by detaching $v$ from some cycles in $H_{1}, \ldots, H_{k}$ so that $\left|V\left(H_{i}\right)\right|$ remains at least two for all $i=1, \ldots, k$.

Proof: Recall that $N \neq \emptyset$. If $|N| \geq 2$, the claim is obvious since each of $H_{1}, \cdots, H_{k}$ is incident to all vertices in $N$. Hence suppose that $|N|=1$, and let $x$ be the unique vertex in $N$. Then all arcs in $M^{\prime}$ are incident to $x$ since otherwise Operation 1 or 2 would be applicable to some vertex in $V-x$. In other words, it hold $\left|M^{\prime}\right|=d^{-}\left(x ; D_{M^{\prime}}\right)+$ $d^{+}\left(v ; D_{M^{\prime}}\right)=b^{-}(x)+b^{+}(x)-2 k$. Recall that $\sum_{v \in V-x} b^{+}(v) \geq b^{-}(x)$ and $\sum_{v \in V-x} b^{-}(v) \geq$ $b^{+}(x)$ hold by the assumption that perfect $\left(b^{-}, b^{+}\right)$-matchings exist. Now assume that we have converted some excess vertices in $D_{A^{\prime}}$ into non-excess vertices by detaching them from some of $H_{1}, \ldots, H_{k}$ while keeping $\left|V\left(H_{i}\right)\right| \geq 2, i=1, \ldots, k$, and yet an excess vertex $y \in V-x$ remains. Then there remains a cycles $H_{i}$ with $\left|V\left(H_{i}\right)\right|>2$ because

$$
\begin{aligned}
\sum_{1 \leq i \leq k}\left|V\left(H_{i}\right)\right|= & \sum_{v \in V} d^{-}\left(v ; D_{H_{1} \cup \cdots \cup H_{k}}\right)=\sum_{v \in V} d^{-}\left(v ; D_{E^{\prime}}\right)-\left|M^{\prime}\right| \\
& >\sum_{v \in V-\{x\}} b^{-}(v)+d^{-}\left(x ; D_{E^{\prime}}\right)-\left|M^{\prime}\right| \geq b^{+}(x)+b^{-}(x)-\left|M^{\prime}\right| \geq 2 k .
\end{aligned}
$$

Hence we can detach $y$ from such $H_{i}$, implying the claim also for $|N|=1$.

In the following, we let $H_{i}^{\prime}$ denote $H_{i}$ after Phase 2 , and $H_{i}$ denote the original Hamiltonian cycle for $i=1, \ldots, k$ in order to avoid the ambiguity. Moreover let $A^{\prime \prime}=$ $M^{\prime} \cup H_{1}^{\prime} \cup \cdots \cup H_{k}^{\prime}$. Our algorithm outputs $D_{A^{\prime \prime}}$ as a solution. 
Input: A vertex set $V$, in- and out-degree specification $b^{-}, b^{+}: V \rightarrow \mathbb{Z}_{+}$, a symmetric metric arc cost $c: V \times V \rightarrow \mathbb{Q}_{+}$, and a positive integer $k$

Output: A $k$-arc-connected perfect $\left(b^{-}, b^{+}\right)$-matching or "INFEASIBLE"

1: if $\sum_{v \in V} b^{-}(v) \neq \sum_{v \in V} b^{+}(v), \exists v: b^{-}(v)>\sum_{u \in V-v} b^{+}(u), \exists v: b^{+}(v)>\sum_{u \in V-v} b^{-}(u)$, $\exists v: k>b^{-}(v)$, or $\exists v: k>b^{+}(v)$ then

2: Output "INFEASIBLE" and halt

3: end if;

4: Compute a minimum cost perfect $\left(b^{-}, b^{+}\right)$-matching $D_{M}$;

5: Compute a Hamiltonian cycle $D_{H}$ on $V$ by Christofides' algorithm; Let $H_{1}, \ldots, H_{k}$ be $k$ copies of $H$;

\# Phase 1

6: $M^{\prime}:=M$;

7: while Operation 1 or 2 is applicable to a vertex $v \in V$

with $d^{-}\left(v ; D_{M^{\prime} \cup H_{1} \cup \cdots \cup H_{k}}\right)>b^{-}(v)$ do

8: if $\exists\{x v, v y\} \subseteq M^{\prime}$ such that $x \neq y$ then

9: $\quad M^{\prime}:=\left(M^{\prime}-\{x v, v y\}\right) \cup\{x y\} \quad$ \# Operation 1

10: $\quad$ else if $\exists\{x v, v x\} \subseteq M^{\prime}$ such that $d^{-}\left(x ; D_{M^{\prime} \cup H_{1} \cup \cdots \cup H_{k}}\right)>b^{-}(x)$ then

11: $\quad M^{\prime}:=M^{\prime}-\{x v, v x\} \quad$ \# Operation 2

12: $\quad$ end if

13: end while;

\# Phase 2

14: $H_{i}^{\prime}:=H_{i}$ for each $i=1, \ldots, k$;

15: while $\exists v \in V$ with $d^{-}\left(v ; D_{M^{\prime} \cup H_{1}^{\prime} \cup \cdots \cup H_{k}^{\prime}}\right)>b^{-}(v)$ do

16: $\quad$ Detach $v$ from $H_{i}^{\prime}$ with $V\left(H_{i}^{\prime}\right)>2$

17: end while;

18: $A^{\prime \prime}:=M^{\prime} \cup H_{1}^{\prime} \cup \cdots \cup H_{k}^{\prime}$;

19: Output $D_{A^{\prime \prime}}$

Let OPT denote the optimal cost of $k$-ACMDS. We can show that $D_{A^{\prime \prime}}$ is $k$-arcconnected, $c(M) \leq \mathrm{OPT}$ and $c\left(H_{i}\right) \leq 1.5 \mathrm{OPT} / k$ for $1 \leq i \leq k$, similarly for $\operatorname{UNDIRECT}(k)$ although we leave the proof to the readers. As a conclusion, we have the following theorem.

Theorem 6 Algorithm $\operatorname{DIRECT}(k)$ is a 2.5-approximation algorithm for $k$-ACMDS.

$\operatorname{Algorithm} \operatorname{DIRECT}(k)$ always outputs a solution when there exists a perfect $\left(b^{-}, b^{+}\right)$matching and $b^{-}(v) \geq k, b^{+}(v) \geq k$ for all $v \in V$. This fact and Theorem 5 implies the following corollary.

Corollary 2 For $k \geq 1$, there exists a $k$-arc-connected perfect $\left(b^{-}, b^{+}\right)$-matching if and only if $\sum_{v \in V} b^{-}(v)=\sum_{v \in V} b^{+}(v), k \leq b^{-}(v) \leq \sum_{u \in V-v} b^{+}(u)$ for each $v \in V$, and $k \leq b^{+}(v) \leq \sum_{u \in V-v} b^{-}(u)$ for each $v \in V$. 


\section{Uniform degree specification}

In this section, we show that the approximation factor of our algorithms can be improved when $b(v)=\ell$ in $k$-ECMDS or $b^{-}(v)=b^{+}(v)=\ell$ in $k$-ACMDS for all $v \in V$ with some integer $\ell \geq k$.

We call a perfect $b$-matching (resp., a perfect $\left(b^{-}, b^{+}\right)$-matching) $M \ell$-regular if $b(v)=\ell$ (resp., $b^{-}(v)=b^{+}(v)=\ell$ ) for all $v \in V$.

Lemma 1 Assume that $b^{-}(v)=b^{+}(v)=\ell$ for all $v \in V$ and an $\ell$-regular digraph exists. Let OPT denote the optimal cost of $k-A C M D S$. Then there exists an $(\ell-m)$-regular digraph $D_{R}$ with $c(R) \leq \frac{\ell-m}{\ell} O P T$ for an arbitrary non-negative integer $m \leq \ell$.

Proof: Let $A$ denote an optimal arc set of $k$-ACMDS. As seen in Section 3, digraph $D_{A}$ corresponds to the bipartite undirected graph $\left(V^{-}, V^{+}, E\right)$, which is a $\ell$-regular. A theorem derived from Frobenius' theorem tells that every $\ell$-regular bipartite graph can be decomposed into $\ell$ graphs each of which is 1-regular [11]. Let $R$ be the set of $\operatorname{arcs}$ corresponding to edges in least cost $\ell-m$ graphs of them. Then $R$ is $(\ell-m)$-regular and $c(R) \leq \frac{\ell-m}{\ell} c(A)$, as required.

The union of an $(\ell-k)$-regular digraph and $k$ Hamiltonian cycles are obviously feasible to $k$-ACMDS if $b^{-}(v)=b^{+}(v)=\ell, v \in V$. Therefore we can derive the following theorem.

Theorem 7 If $b^{-}(v)=b^{+}(v)=\ell$ for all $v \in V$, then $k$-ACMDS is approximable within a factor of $1.5+\frac{\ell-k}{\ell}$.

Next, we consider $k$-ECMDS.

Lemma 2 Assume that $b(v)=\ell$ for all $v \in V$ and an $\ell$-regular graph exists. Let OPT denote the optimal cost of $k-E C M D S$. Then there exists an $(\ell-2 m)$-regular graph $G_{R}$ such that $c(R) \leq \frac{\ell-2 m}{\ell} O P T$ if $\ell$ is even, and $c(R) \leq\left(\frac{\ell-2 m-1}{\ell}+\frac{1}{k}\right) O P T$ if $\ell$ is odd for an arbitrary non-negative integer $m$ with $2 m \leq \ell$.

Proof: Let $E$ denote an optimal edge set of $k$-ECMDS. First suppose that $\ell$ is even. Then $E$ can be oriented into an $\operatorname{arc}$ set $A$ such that $D_{A}$ is $\ell / 2$-regular. Let $c^{\prime}$ be an $\operatorname{arc}$ cost on $A$ naturally defined from $c$ (i.e., $c^{\prime}(a)=c(e)$ if $a \in A$ corresponds to $e \in E$ ). As in the proof of Lemma 1 , we can obtain an $(\ell / 2-m)$-regular digraph $R^{\prime}$ with $c^{\prime}\left(R^{\prime}\right) \leq \frac{\ell / 2-m}{\ell / 2} c^{\prime}(A)$. Let $R$ be an edge set corresponding to $R^{\prime}$. Then clearly $G_{R}$ is $(\ell-2 m)$-regular and $c(R) \leq \frac{\ell / 2-m}{\ell / 2} c(E)$, as required.

Next, suppose that $\ell$ is odd. Let $2 E$ denote the edge set obtained by duplicating each edge in $E$. Then $G_{2 E}$ is $2 \ell$-regular. By the above argument about the case of $\ell$ is even, we can obtain an $(\ell-2 m-1)$-regular graph $G_{F}$ such that $c(F) \leq \frac{\ell-2 m-1}{2 \ell} c(2 E)=\frac{\ell-2 m-1}{\ell} c(E)$ (Notice that $\ell-2 m-1$ is even). Let $M$ be a minimum cost 1-regular graph. Notice that such $M$ exists since $|V|$ is even by the existence of an $\ell$-regular graph with odd $\ell$. Since the minimum cost of Hamiltonian cycles spanning all vertices is at most $2 c(E) / k$ as shown in the proof of Claim 6 , we can see that $c(M) \leq c(E) / k$. Let $R=F \cup M$. Then $G_{R}$ is $(\ell-2 m)$-regular and $c(R)=c(F)+c(M) \leq\left(\frac{\ell-2 m-1}{\ell}+\frac{1}{k}\right) c(E)$, as required. 
Let $k^{\prime}=\lceil k / 2\rceil$. The union of an $\left(\ell-2 k^{\prime}\right)$-regular graph and $2 k^{\prime}$ Hamiltonian cycles are obviously feasible to $k$-ECMDS if $b(v)=\ell, v \in V$. Therefore we can derive the following theorem.

Theorem 8 If $b(v)=\ell$ for all $v \in V$, then $k-E C M D S$ is approximable within a factor of $\frac{\ell-2 k^{\prime}}{\ell}+3 \frac{k^{\prime}}{k}$ if $\ell$ is even, and $\frac{\left(\ell-2 k^{\prime}-1\right)}{\ell}+\frac{1+3 k^{\prime}}{k}$ if $\ell$ is odd, where $k^{\prime}=\lceil k / 2\rceil$.

Recall that metric TSP can be formulated as $k$-ECMDS with $b(v)=2, v \in V$ and $k=2$. Theorem 8 indicates that this case can be approximated within 1.5 as Christofides' algorithm.

\section{Application for $(m, n)$-VRP}

In this section, we consider the problem $(m, n)$-VRP. The formal definition of this problem is as follows. An instance of $(m, n)$-VRP consists of a vertex set $V$ containing a special vertex $s$, a metric edge cost $c:\left(\begin{array}{l}V \\ 2\end{array}\right) \rightarrow \mathbb{Q}_{+}$, and two non-negative integers $m$ and $n$. The objective is to find a minimum cost set of $m$ cycles, each containing $s$, such that each vertex in $V-s$ is contained in exactly $n$ of those cycles. We can assume without loss of generality that $n \leq m \leq n(|V|-1)$ since otherwise the instance is clearly infeasible.

An example of applying the $(m, n)$-VRP is the schedule of garbage collection. Let us consider the case in which a garbage collecting truck must visit each city on $n$ of 5 weekdays in a week. A solution of $(5, n)$-VRP gives a schedule of this truck minimizing total length of routes.

Each solution to $(m, n)$-VRP is obviously feasible to $2 n$-ECMDS with $b(s)=2 m$ and $b(v)=2 n$ for $v \in V-s$ (Hence the optimal value of $2 n$-ECMDS with such $b$ is at most that of $(m, n)$-VRP). However, the opposite direction does not hold as an example in Figure 5. Nevertheless we can see that algorithm UNDIRECT $(2 n)$ outputs a feasible solution for $(m, n)$-VRP.

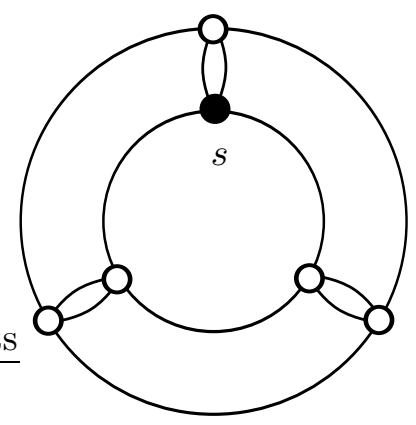

Figure 4: A solution to 4-ECMDS with $b(v)=4, v \in V$, that is not feasible to $(2,2)$-VRP

Theorem 9 Let $b(s)=2 m, b(v)=2 n$ for each $v \in V-s$ and $k=2 n$. Then algorithm $\mathrm{UNDIRECT}(k)$ outputs a 2.5-approximate solution to $(m, n)$-VRP.

Proof: The solution given by algorithm UNDIRECT $(k)$ consists of edge set $M^{\prime}$ and cycles $H_{1}^{\prime}, \ldots, H_{n}^{\prime}$. In what follows, we see that this solution is feasible to $(m, n)$-VRP. 
Let us consider the moment after Phase 1 , and define $E^{\prime}, M^{\prime}$ and $H_{1}^{\prime}, \ldots, H_{k^{\prime}}^{\prime}$ as in Section 2. Since $k=2 n$ is even, there exists no strict pair. Hence at least one end vertex of each edge in $M^{\prime}$ is a non-excess vertex. Let $v$ be such a vertex. Then $b(v)=$ $d\left(v ; G_{E^{\prime}}\right)>d\left(v ; G_{H_{1} \cup \cdots \cup H_{n}}\right)=2 n$ (Recall that each non-excess vertex is covered by all of $\left.H_{1}, \ldots, H_{n}\right)$. However, a vertex of degree more than $2 n$ is only $s$ since $b(u)=2 n$ for each $u \in V-s$. Hence we can see that (i) $s$ is a non-excess vertex after Phase 1 , and (ii) one end vertex of each in $M^{\prime}$ is $s$. Condition (i) implies that each of $H_{1}^{\prime}, \ldots, H_{n}^{\prime}$ covers $s$. Condition (ii) indicates that edges between $s$ and a vertex $v \in V-s$ forms $d\left(v ; M^{\prime}\right) / 2$ cycles whose vertex sets are $\{s, v\}$ because $d\left(v ; M^{\prime}\right)$ is even. Therefore, combining the fact that $d\left(v ; G_{M^{\prime} \cup H_{1}^{\prime} \cup \cdots \cup H_{n}^{\prime}}\right)=b(v)$ for all $v \in V$, these shows that $\operatorname{UNDIRECT}(k)$ outputs a feasible solution to $(m, n)$-VRP.

The approximation factor can be improved as follows.

Theorem 10 Problem $(m, n)-V R P$ can be approximated within a factor of $1.5+\frac{m-n}{m}$.

Proof: Let $b(s)=2 m, b(v)=2 n$ for each $v \in V-s$ and $k=2 n$. Moreover, let $E$ be an optimal solution for $(m, n)$-VRP, and $F$ be the set of edges contained by $m-n$ cycles in $G_{E}$ of least cost. Then it holds that $d\left(s ; G_{F}\right)=2 m-2 n$ and $d\left(v ; G_{F}\right) \leq 2 n$ for $v \in V-s$. Besides this, we have $c(F) \leq \frac{m-n}{m} c(E)$ by the definition of $F$.

Now we let $V-s=\left\{v_{1}, \ldots, v_{|V|-1}\right\}$ so that $c\left(s v_{1}\right) \leq c\left(s v_{2}\right) \leq \cdots \leq c\left(s v_{|V|-1}\right)$. Moreover we define $R$ as an edge set which consists of $2 n$ edges $s v_{i}$ for each $i=1, \ldots, p$ and $2 m-2 n(p+1)$ edges $s v_{p+1}$, where $p=\lfloor(m-n) / n\rfloor$. Then it is clear that $R$ is a minimum cost edge set such that $d\left(s ; G_{R}\right)=2 n p+2 m-2 n(p+1)=2 m-2 n$ and $d\left(v ; G_{R}\right) \leq 2 n$ for all $v \in V-s$. This implies that $c(R) \leq c(F) \leq \frac{m-n}{m} c(E)$.

By using $R$ instead of $M$ in $\operatorname{UNDIRECT}(k)$, we can obtain a feasible solution to $k$ ECMDS. As in Theorem 9, this solution is also feasible to $(m, n)$-VRP. Moreover the cost of the solution is at most $c\left(H_{1}\right)+\cdots+c\left(H_{k^{\prime}}\right)+c(R) \leq\left(1.5+\frac{m-n}{m}\right) c(E)$, which completes the proof.

\section{Concluding Remarks}

We note that some cases of $k$-ECMDS/ $k$-ACMDS remain open. One is 1-ECMDS with $b(v)=1$ for some $v \in V$. Our algorithm cannot deal with this case, because detaching the vertices in a strict pair from the same Hamiltonian cycle in Phase 2 may lose the connectivity. Also a key problem for approximating 1-ECMDS would be to find a minimum cost spanning tree such that $d(v) \leq b(v), v \in V$ for a given $b: V \rightarrow \mathbb{Z}_{+}$. However, no constant factor approximation algorithm is known to this problem if $b(v)=1$ for some $v \in V$, although it can be approximated within a constant factor of 2 if $b(v) \geq 2$ for all $v \in V$ [1]. Another interesting open problem is a generalization of $k$-ECMDS (resp., $k$-ACMDS) in which the $k$-edge-connectivity (resp., $k$-arc-connectivity) requirement is replaced by a local-edge-connectivity requirement.

It is also valuable to characterize the feasible solutions to $(m, n)$-VRP. In Section 5 , we noted that specifying the edge-connectivity and the degree of each vertex is not enough for this although our algorithm always outputs a feasible solution to $(m, n)$-VRP. Moreover, it 
is interesting to study a further generalizatoin of $(m, n)$-VRP in which the number $b(v) / 2$ of cycles containing each vertex $v$ is not uniform.

\section{Acknowledgement}

This research was partially supported by the Scientific Grant-in-Aid from Ministry of Education, Culture, Sports, Science and Technology of Japan.

\section{References}

[1] S. P. Fekete, S. Khuller, M. Klemmstein, B. Raghavachari, N. Young, A network-flow technique for finding low-weight bounded-degree spanning trees, Journal of Algorithms 24 (1997) 310-324.

[2] A. Frank, Augmenting graphs to meet edge-connectivity requirements, SIAM Journal on Discrete Mathematics 5 (1992) 25-53.

[3] A. Frank, On a theorem of Mader, Discrete Mathematics 191 (1992) 49-57.

[4] G. N. Frederickson, M. S. Hecht, C. E. Kim, Approximation algorithms for some routing problems, SIAM Journal of Computing 7 (1978) 178-193.

[5] T. Fukunaga, H. Nagamochi, Approximating minimum cost multigraphs of specified edge-connectivity under degree bounds, Proceedings of the 9th Japan-Korea Joint Workshop on Algorithm and Computation (2006) 25-32.

[6] T. Fukunaga, H. Nagamochi, Approximating a generalization of metric TSP, IEICE Transactions on Information and Systems, to appear.

[7] M. X. Goemans, D. J. Bertsimas, Survivable networks, linear programming relaxations and the parsimonious property, Mathematical Programming 60 (1993) 145166.

[8] M. X. Goemans, D. P. Williamson, The primal-dual method for approximation algorithms and its application to network design problems, PWS, 1997, Ch. 4, pp. $144-191$.

[9] E. L. Lawler, J. K. Lenstra, A. H. G. Rinnooy Kan, D. B. Shmoys (Eds.), The Traveling Salesman Problem: A Guided Tour of Combinatorial Optimization, John Wiley \& Sons, 1985.

[10] W. Mader, A reduction method for edge-connectivity in graphs, Annals of Discrete Mathematics 3 (1978) 145-164.

[11] A. Schrijver, Combinatorial Optimization: Polyhedra and Efficiency, Springer, 2003.

[12] V. Vazirani, Approximation Algorithm, Springer, 2001.

[13] L. A. Wolsey, Heuristic analysis, linear programming and branch and bound, Mathematical Programming Study 13 (1980) 121-134. 